Journal of Mathematics and Statistics 5 (3):206-214, 2009

ISSN 1549-3644

(C) 2009 Science Publications

\title{
An Optimal Control Approach to Inventory-Production Systems with Weibull Distributed Deterioration
}

\author{
Md. Azizul Baten and Anton Abdulbasah Kamil \\ School of Distance Education, University Sains Malaysia \\ 11800 USM, Penang, Malaysia
}

\begin{abstract}
Problem statement: We studied the inventory-production system with two-parameter Weibull distributed deterioration items. Approach: The inventory model was developed as linear optimal control problem and by the Pontryagin maximum principle, the optimal control problem was solved analytically to obtain the optimal solution of the problem. Results: It was then illustrated with the help of an example. By the principle of optimality we also established the Riccati based solution of the Hamilton-Jacobi-Bellman (HJB) equation associated with this control problem. Conclusion: As an application to quadratic control theory we showed an optimal control policy to exist from the optimality conditions in the HJB equation.
\end{abstract}

Key words: Inventory-production system, Weibull distributed deterioration, linear quadratic regulator, principle of optimality, optimal control.

\section{INTRODUCTION}

Many management science applications involve the control of dynamic systems, i.e., systems that evolve over time called continuous time systems or discretetime systems depending on whether time varies continuously or discretely which is a rich research area $^{[22]}$. We are especially interested in the application of optimal control theory to the production planning problem. Inventory-Production system consists of a manufacturing plant and a finished goods warehouse to store those products which are manufactured but not immediately sold. The advantages of having products in inventory are: First they are immediately available to meet demand; second, by using the warehouse to store excess production during low demand periods to be available for sale during high demand periods. Typically, the firm has to balance the high production costs and find the quantity it should produce in order to keep the total cost at a minimum.

Now a days the optimal control theory has been applied to different inventory-production control problems where researchers are involved to analyze the effect of deterioration and the variations in the demand rate with time in logistics. The model of inventoryproduction system considered the inventory depletion not only by demand but also by item's deterioration ${ }^{[10]}$. These types of problems have been studied by several researchers to determine the optimum order quantity for different demand patterns ${ }^{[1,2,14]}$. The model was presented for deteriorating items with time proportional demand ${ }^{[14]}$ whereas a heuristic model was developed that allowed the variation in both replenishment-cycle length and the size of the order ${ }^{[2]}$. A linear quadratic regulator problem was used with known disturbance to an inventory-production system with items that deteriorate at a known constant rate ${ }^{[1]}$. They used a first-order model to represent the inventory system in deriving the optimal production policy. Importance of items of deteriorating in inventory modeling is now widely acknowledged, as shown by ${ }^{[13,23]}$. A number of studies have been done with the assumption that the deterioration rate follows the Weibull distribution ${ }^{[5,6,11,12,20,25]}$. The assumption of the constant deterioration rate was relaxed ${ }^{[7]}$ using a two-parameter Weibull distribution to represent the distribution of time to deterioration. This model was further generalized ${ }^{[19]}$ taking a three-parameter Weibull distribution. A twoparameter Weibull distribution deterioration is adopted to develop an inventory model with a finite rate of replenishment ${ }^{[18]}$.

In general, in formulating inventory models, two factors of the problem have been of growing interest to the researchers, one being the deterioration of items and the other being the variation in the demand rate with time. This study develops an optimal control model and utilizes the optimal control theory to obtain optimal production policy for inventory production systems

Corresponding Author: Md. Azizul Baten, School of Distance Education, University Sains Malaysia, 11800 USM, Penang, Malaysia. 
where the novelty we take into consideration in our research is that the time of deterioration is a random variable followed by the two-parameter Weibull distribution. This distribution can be used to model either increasing or decreasing rate of deterioration, according to the choice of the parameters. The probability density function of a two-parameter Weibull distribution is given by:

$$
g(t)=\eta \gamma t^{\gamma-1} e^{-\eta t^{\gamma}}, \quad t>0
$$

where, $\eta>0$ is the scale parameter, $\gamma>0$ is the shape parameter. The probability distribution function is:

$$
G(t)=1-e^{-\eta t^{\gamma}}, \quad t>0
$$

The instantaneous rate of deterioration of the onhand inventory is given by:

$$
\mathrm{A}(\mathrm{t})=\frac{\mathrm{g}(\mathrm{t})}{1-\mathrm{G}(\mathrm{t})}=\eta \gamma \mathrm{t}^{\gamma-1}, \quad \mathrm{t}>0
$$

Applications of optimization methods to production and inventory problems date back at least to the classical economic order quantity model. Some references that apply control theory to production and inventory problems are ar, $^{[4,8,15,16,19,24]}$.

In the present study, we assume that timedependence of the demand rate. Deterioration rate is assumed to follow a two-parameter Weibull distribution. The purpose of the study is to give an optimal production policy which minimizes the cost for inventory production systems where items are deteriorating with a Weibull -distribution. To establish the Riccati based solution form to the Hamilton JacobiBellman (in short, HJB) equation associated with the optimal control problem is also of our interest. Finally an attempt has been made to give an optimal control policy from the optimality condition in the HJB equation.

\section{MATERIALS AND METHODS}

To build our optimal control model, we consider that a firm can manufactures a certain product, selling some and stocking the rest in a warehouse. We assume that the demand rate varies with time and the firm has set an inventory goal level and production goal rate. We also assume that the firm has no shortage, the instantaneous rate of deterioration of the on-hand inventory follows the two-parameter Weibull distribution and the production is continuous.
Since our objective is to minimize the setup and the inventory costs, the objective function can be expressed as the quadratic form:

$$
\operatorname{minimize} \quad J=\frac{1}{2} \int_{0}^{T}\left\{h[x(t)-\hat{x}(t)]^{2}+C[u(t)-\hat{u}(t)]^{2}\right\} d t
$$

The interpretation of this objective function is that we want to keep the inventory $\mathrm{x}$ as close as possible to its goal $\hat{x}$ and also keep the production rate $u$ as close to its goal level $\hat{u}$. The quadratic terms $\mathrm{h}[\mathrm{x}(\mathrm{t})-\hat{\mathrm{x}}(\mathrm{t})]^{2}$; and $\mathrm{C}[\mathrm{u}(\mathrm{t})-\hat{\mathrm{u}}(\mathrm{t})]^{2}$ impose 'penalties' for having either $\mathrm{x}$ or $\mathrm{u}$ not being close to its corresponding goal level. The dynamics of the state equation of this dynamic model which says that the inventory at time $t$ is increased by the production rate and decreased by the demand rate and the instantaneous rate of deterioration $\eta \gamma \mathrm{t}^{\gamma-1}$ of Weibull distribution can be written as according to:

$$
d x(t)=\left[u(t)-y(t)-\eta \gamma t^{\gamma-1} x(t)\right] d t \quad x(0)=x, \quad x>0
$$

Where:

$\mathrm{x}(\mathrm{t})=$ The inventory level in the warehouse at any instant of time $t \in[0, \mathrm{~T}]$

$\hat{\mathrm{x}}(\mathrm{t}) \quad=$ An inventory goal level what is set by the firm taking into consideration the available storage space,

$\mathrm{h} \geq 0 \quad=$ The inventory holding cost incurred for the inventory level to deviate from its goal

$\mathrm{u}(\mathrm{t}) \geq 0=$ The firm manufactured units of the production rate at any instant of time $t \in[0, \mathrm{~T}]$

$\hat{\mathrm{u}}(\mathrm{t})=$ The production goal rate

$\mathrm{C}>0=$ The unit cost incurred for the production rate to deviate from its goal

$\mathrm{y}(\mathrm{t})=$ The demand rate

$\mathrm{T}>0=$ Represents the fixed length of the planning horizon

The methodologies applied in this study are as follows: Pontryagin maximum principle ${ }^{[21]}$ and the principle of optimality ${ }^{[3]}$. Pontryagin maximum principle is used to solve the optimal control problem analytically and to obtain the optimal solution of this problem. The principle of optimality is also used to establish the Riccati based solution of the HamiltonJacobi-Bellman (HJB) equation associated with this control problem.

\section{RESULTS AND DISCUSSION}

Development of the optimal control model: By the virtue of (2) the instantaneous state of the inventory 
level $x(t)$ at any time $t$ is governed by the differential equation:

$$
\begin{aligned}
& \frac{d x(t)}{d t}+\eta \gamma t^{\gamma-1} x(t)=u(t)-y(t), 0 \leq t \leq t_{1}, \\
& x(0)=x \text { and } x\left(t_{1}\right)=0
\end{aligned}
$$

This is a linear ordinary differential equation of first order and its integrating factor is:

$$
=\exp \left\{\eta \gamma \int \mathrm{t}^{\gamma-1} \mathrm{dt}\right\}=\exp \left\{\eta \mathrm{t}^{\gamma}\right\}
$$

Multiplying both sides of (3) by $\exp \left\{\eta \mathrm{t}^{\gamma}\right\}$ and then integrating over $[0, t]$, we have:

$$
\begin{gathered}
\mathrm{x}(\mathrm{t}) \exp \left\{\eta \mathrm{t}^{\gamma}\right\}-\mathrm{x}(0)=-\int_{0}^{\mathrm{t}}[\mathrm{y}(\mathrm{t})-\mathrm{u}(\mathrm{t})] \exp \left\{\eta \mathrm{t}^{\gamma}\right\} \mathrm{dt}, \\
0 \leq \mathrm{t} \leq \mathrm{t}_{1}
\end{gathered}
$$

Substituting this value of $\mathrm{x}(0)$ in (4), we obtain the instantaneous level of inventory at any time $t \in[0, T]$ is given by:

$$
\mathrm{x}(\mathrm{t})=\frac{\int_{0}^{\mathrm{t}_{1}}[\mathrm{y}(\mathrm{t})-\mathrm{u}(\mathrm{t})] \exp \left\{\eta \mathrm{t}^{\gamma}\right\} \mathrm{dt}-\int_{0}^{\mathrm{t}}[\mathrm{y}(\mathrm{t})-\mathrm{u}(\mathrm{t})] \exp \left\{\eta \mathrm{t}^{\gamma}\right\} \mathrm{dt}}{\exp \left\{\eta \mathrm{t}^{\gamma}\right\}},
$$

In order to develop the optimal control model, we start by defining the variables $z(t), k(t)$ and $v(t)$ such that

$$
\begin{aligned}
& z(t)=x(t)-\hat{x}(t), k(t)=u(t)-\hat{u}(t) \\
& v(t)=\hat{u}(t)-y(t)-\eta \gamma \gamma^{\gamma-1} \hat{x}(t)
\end{aligned}
$$

By adding and subtracting the last term $\eta \gamma \mathrm{t}^{\gamma-1} \hat{\mathrm{x}}(\mathrm{t})$ from the right hand side of Eq. 6 and rearranging the terms we have:

$$
d z(t)=\left[-\eta \gamma t^{\gamma-1}(x(t)-\hat{x}(t))+u(t)-y(t)-\eta \gamma t^{\gamma-1} \hat{x}(t)\right] d t
$$

Hence:

$$
d z(t)=\left[-\eta \gamma t^{\gamma-1} z(t)+u(t)-y(t)-\eta \gamma t^{\gamma-1} \hat{x}(t)\right] d t
$$

Now substituting (5) and (6) in (7) yields:

$\mathrm{dz}(\mathrm{t})=\left[-\eta \gamma \mathrm{t}^{\gamma-1} \mathrm{z}(\mathrm{t})+\mathrm{k}(\mathrm{t})+\mathrm{v}(\mathrm{t})\right] \mathrm{dt}$.
The optimal control model becomes:

$\operatorname{minimize} \quad \mathrm{J}=\int_{0}^{\mathrm{T}}\left\{\mathrm{h}\left[\mathrm{z}(\mathrm{t})^{2}\right]+\mathrm{C}\left[\mathrm{k}(\mathrm{t})^{2}\right]\right\} \mathrm{dt}$

subject to an ordinary differential equation:

$\mathrm{dz}(\mathrm{t})=\left[-\eta \gamma \mathrm{t}^{\gamma-1} \mathrm{z}(\mathrm{t})+\mathrm{k}(\mathrm{t})+\mathrm{v}(\mathrm{t})\right] \mathrm{dt} \quad \mathrm{z}(0)=\mathrm{z}, \quad \mathrm{z}>0$

This form is a standard Linear Quadratic Regulator (LQR) problem with known disturbance $\mathrm{v}(\mathrm{t})$ defined in (6). The general form of this LQR optimal control problem for a finite time horizon $[0, \mathrm{~T}]$ is the following:

$\operatorname{minimize} \quad J=\frac{1}{2} \int_{0}^{\mathrm{T}}\left\{\mathrm{z}^{\mathrm{T}}(\mathrm{t}) \mathrm{Q}(\mathrm{t}) \mathrm{z}(\mathrm{t})+\mathrm{k}^{\mathrm{T}}(\mathrm{t}) \mathrm{R}(\mathrm{t}) \mathrm{k}(\mathrm{t})\right\} \mathrm{dt}$

subject to an ordinary differential equation:

$\mathrm{dz}(\mathrm{t})=\left[\mathrm{A}_{1}(\mathrm{t}) \mathrm{z}(\mathrm{t})+\mathrm{B}_{1}(\mathrm{t}) \mathrm{k}(\mathrm{t})+\mathrm{v}(\mathrm{t})\right] \mathrm{dt} \quad \mathrm{z}(0)=\mathrm{z}, \mathrm{z}>0$

Where:

$\mathrm{Q}(\mathrm{t})$ and $\mathrm{R}(\mathrm{t}) \quad=$ Real symmetric positive semidefinite matrices of appropriate dimension

$\mathrm{A}_{1}(\mathrm{t})$ and $\mathrm{B}_{1}(\mathrm{t})=$ The system dynamics matrices

$\mathrm{T}>0 \quad$ fixed

Solution to the optimal control problem:

Solution by pontryagin maximum principle: In order to find extremals for this optimal control problem (11) and (12), we apply Pontryagin maximum principle to form the Hamiltonian as:

$$
\mathrm{H}(\mathrm{x}, \phi, \mathrm{k}, \mathrm{t})=\frac{1}{2}\left[\begin{array}{l}
\left\{\mathrm{z}^{\mathrm{T}}(\mathrm{t}) \mathrm{Q}(\mathrm{t}) \mathrm{z}(\mathrm{t})+\mathrm{k}^{\mathrm{T}}(\mathrm{t}) \mathrm{R}(\mathrm{t}) \mathrm{k}(\mathrm{t})\right\} \\
+\phi^{\mathrm{T}}(\mathrm{t})\left\{\mathrm{A}_{1}(\mathrm{t}) \mathrm{z}(\mathrm{t})+\mathrm{B}_{1}(\mathrm{t}) \mathrm{k}(\mathrm{t})+\mathrm{v}(\mathrm{t})\right\}
\end{array}\right] .
$$

Then the necessary conditions of optimality give the co-states equations as:

$$
\begin{aligned}
\mathrm{d} \phi^{\mathrm{T}}(\mathrm{t}) & =-\mathrm{H}_{\mathrm{z}} \mathrm{dt}=\left[-\mathrm{z}^{\mathrm{T}}(\mathrm{t}) \mathrm{Q}(\mathrm{t})-\mathrm{A}_{1}(\mathrm{t}) \phi^{\mathrm{T}}(\mathrm{t})\right] \mathrm{dt} \\
\mathrm{d} \phi(\mathrm{t}) & =\left[-\mathrm{Q}(\mathrm{t}) \mathrm{z}(\mathrm{t})-\mathrm{A}_{1}^{\mathrm{T}}(\mathrm{t}) \phi(\mathrm{t})\right] \mathrm{dt}
\end{aligned}
$$

The extremal control vector is given by:

$$
\mathrm{H}_{\mathrm{k}}=\mathrm{R}(\mathrm{t}) \mathrm{k}(\mathrm{t})+\mathrm{B}_{1}^{\mathrm{T}}(\mathrm{t}) \phi(\mathrm{t})=0
$$

from which we have:

$\mathrm{k}(\mathrm{t})=-\mathrm{R}^{-1}(\mathrm{t}) \mathrm{B}_{1}^{\mathrm{T}}(\mathrm{t}) \phi(\mathrm{t})$ 
because $\mathrm{R}$ is nonsingular. Then from (12), the extremal state vector satisfies:

$d z(t)=\left[A_{1}(t) z(t)-B_{1}(t) R^{-1}(t) B_{1}^{T}(t) \phi(t)\right] d t$

Now by combining (13) and (15), the state-costate equations can be written in matrix as follows:

$$
\left(\begin{array}{l}
\mathrm{dz}(\mathrm{t}) \\
\mathrm{d} \phi(\mathrm{t})
\end{array}\right)=\left(\begin{array}{cc}
\mathrm{A}_{1}(\mathrm{t}) & -\mathrm{B}_{1}(\mathrm{t}) \mathrm{R}^{-1}(\mathrm{t}) \mathrm{B}_{1}^{\mathrm{T}}(\mathrm{t}) \\
-\mathrm{Q}(\mathrm{t}) & -\mathrm{A}_{1}^{\mathrm{T}}(\mathrm{t})
\end{array}\right)\left(\begin{array}{l}
\mathrm{z}(\mathrm{t}) \\
\phi(\mathrm{t})
\end{array}\right)
$$

The solution to this system of linear differential equation is of the form:

$$
\left(\begin{array}{l}
\mathrm{z}(\mathrm{T}) \\
\phi(\mathrm{T})
\end{array}\right)=\varphi(\mathrm{T}, \mathrm{t})\left(\begin{array}{l}
\mathrm{z}(\mathrm{t}) \\
\phi(\mathrm{t})
\end{array}\right)
$$

Where:

$$
\varphi(T, t):=\left(\begin{array}{cc}
\mathrm{A}_{1}(\mathrm{t}) & -\mathrm{B}_{1}(\mathrm{t}) \mathrm{R}^{-1}(\mathrm{t}) \mathrm{B}_{1}^{\mathrm{T}}(\mathrm{t}) \\
-\mathrm{Q}(\mathrm{t}) & -\mathrm{A}_{1}^{\mathrm{T}}(\mathrm{t})
\end{array}\right)
$$

is the state transition matrix. Now by the transversality condition $\phi(T)=0$ for LQR problem, we obtain:

$$
z(T)=A_{1}(t) z(t)-B_{1}(t) R^{-1}(t) B_{1}{ }^{T}(t) \phi(t)
$$

and

$$
\phi(T)=-Q(t) z(t)-A_{1}^{T}(t) \phi(t)=0
$$

If $-\mathrm{A}_{1}^{\mathrm{T}}(\mathrm{t})$ is nonsingular for all $\mathrm{t}$ in $[0, \mathrm{~T}]$ then from (16), we have:

$$
\phi(t)=-A_{1}^{T}(t)^{-1} Q(t) z(t)
$$

which establish a linear relationship between $\phi(t)$ and $\mathrm{z}(\mathrm{t})$ :

$\phi(t)=M(t) z(t)$

Where:

$$
M(t)=-A_{1}^{T}(t)^{-1} Q(t)
$$

Then substituting (17) in (14), we obtain the optimal control which is given by a linear feedback law:

$$
k(t)=-R^{-1}(t) B_{1}^{T}(t) M(t) z(t)
$$

That minimizes (12). Now differentiating both sides of (17) and, then by (12) and (19), we have:

$$
\begin{aligned}
\mathrm{d} \phi(\mathrm{t})= & d M(t) z(t)+M(t) d z(t) \\
= & d M(t) z(t)+M(t)\left[A_{1}(t) z(t)+B_{1}(t) k(t)+v(t)\right] \\
= & d M(t) z(t)+M(t) A_{1}(t) z(t)-M(t) B_{1}(t) R^{-1}(t) B_{1} \\
& { }^{T}(t) M(t) z(t)+M(t) v(t)
\end{aligned}
$$

Substituting (17) to (13) we have:

$d \phi(t)=-Q(t) z(t)-A_{1}{ }^{T}(t) M(t) z(t)$

Combining (20) and (21), we obtain:

$\left[\mathrm{dM}(\mathrm{t})+\mathrm{M}(\mathrm{t}) \mathrm{A}_{1}(\mathrm{t})+\mathrm{A}_{1}^{\mathrm{T}}(\mathrm{t}) \mathrm{M}(\mathrm{t})\right.$

$\left.-M(t) B_{1}(t) R^{-1}(t) B_{1}^{T}(t) M(t)+Q(t)\right] z(t)+M(t) v(t)=0$

Since (22) must hold for any value of $z(t)$ and for all $\mathrm{t}$ in $[0, \mathrm{~T}]$ we must have:

$$
\begin{aligned}
d M(t)= & {\left[M(t) B_{1}(t) R^{-1}(t) B_{1}{ }^{T}(t) M(t)-M(t)\left\{A_{1}(t)\right.\right.} \\
& \left.\left.+A_{1}^{T}(t)\right\}-Q(t)\right] d t
\end{aligned}
$$

is called a Riccati equation and $M(t)$ is the Riccati matrix. The boundary conditions are:

$$
\mathrm{z}(0)=0, \quad \phi(0)=0 \quad \text { and } \quad \mathrm{M}(0)=0
$$

By comparing Eq. 9-12, we have:

$$
\mathrm{A}_{1}(\mathrm{t})=-\eta \gamma \mathrm{t}^{\gamma-1}, \quad \mathrm{~B}_{1}=1, \mathrm{Q}=\mathrm{h} \quad \text { and } \quad \mathrm{R}=\mathrm{C}
$$

Then from (18) we obtain:

$$
M(t)=h\left(\eta \gamma t^{\gamma-1}\right)^{-1}
$$

and the optimal control policy (20) becomes:

$$
\mathrm{k}(\mathrm{t})=-\mathrm{hC} \mathrm{C}^{-1}\left(\eta \gamma \mathrm{t}^{\gamma-1}\right)^{-1} \mathrm{z}(\mathrm{t})
$$

Example 1: If we choose:

$$
\mathrm{A}_{1}=-1(\text { by } \eta=1, \gamma=1), \mathrm{B}_{1}=1, \mathrm{Q}=\mathrm{h}=1 \text { and } \mathrm{R}=\mathrm{C}=1
$$

then the optimal control model (11) and (12) becomes over an finite time horizon $[0, \mathrm{~T}]$ :

$$
\operatorname{minimize} \quad \mathrm{J}(\mathrm{k}(\mathrm{t}))=\frac{1}{2} \int_{0}^{\mathrm{T}}\left\{\mathrm{z}^{2}(\mathrm{t})+\mathrm{k}^{2}(\mathrm{t})\right\} \mathrm{dt}
$$


subject to the control system:

$$
\mathrm{dz}(\mathrm{t})=[-\mathrm{z}(\mathrm{t})+\mathrm{k}(\mathrm{t})+\mathrm{v}(\mathrm{t})] \mathrm{dt} \quad \mathrm{z}(0)=\mathrm{z}, \quad \mathrm{z}>0
$$

So the optimal (state) feedback control is given by:

$$
\mathrm{k}(\mathrm{t})=-\mathrm{M}(\mathrm{t}) \mathrm{z}(\mathrm{t})
$$

The Riccati Eq. 23 becomes with the scalar values (chosen above):

$d M(t)-M^{2}(t)-2 M(t)+1=0$ (26):

Now by the method of separation of variables to

$$
d M(t)=\left[M^{2}(t)+2 M(t)-1\right] d t
$$

then:

$$
\frac{1}{2 \sqrt{2}} \int\left(\frac{1}{M(t)+1-\sqrt{2}}-\frac{1}{M(t)+1+\sqrt{2}}\right) d M(t)=t+C_{1}
$$

from which we have:

$\ln \left|\frac{\mathrm{M}(\mathrm{t})+1-\sqrt{2}}{\mathrm{M}(\mathrm{t})+1+\sqrt{2}}\right|=2 \sqrt{2} \mathrm{t}+\mathrm{C}_{1}$

where, $\mathrm{C}_{1}$ is a constant.

Setting $q(t)=M(t)+1$ and substituting to (28) then we have:

$$
\frac{\mathrm{q}(\mathrm{t})-\sqrt{2}}{\mathrm{q}(\mathrm{t})+\sqrt{2}}=\mathrm{Ne}^{2 \sqrt{2} \mathrm{t}}
$$

where, $\mathrm{N}$ is a integration constant. Now by using the boundary condition for $\mathrm{M}(\mathrm{T})=0$ we have:

$$
q(t)=M(T)+1=1=\frac{\sqrt{2}\left(1+\mathrm{Ne}^{2 \sqrt{2} t}\right)}{1-\mathrm{Ne}^{2 \sqrt{2} \mathrm{t}}}
$$

from which $\mathrm{N}=\frac{\sqrt{2}-1}{\sqrt{2}+1} \mathrm{e}^{-2 \sqrt{2} \mathrm{~T}}$ therefore we obtain:

$$
q(t)=\sqrt{2} \frac{1-\frac{\sqrt{2}-1}{\sqrt{2}+1} e^{2 \sqrt{2}(t-T)}}{1+\frac{\sqrt{2}-1}{\sqrt{2}+1} e^{2 \sqrt{2}(t-T)}}
$$

and

$$
\mathrm{M}(\mathrm{T})=\sqrt{2} \frac{\sqrt{2}+1-(\sqrt{2}-1) \mathrm{e}^{2 \sqrt{2}(\mathrm{t}-\mathrm{T})}}{\sqrt{2}+1+(\sqrt{2}-1) \mathrm{e}^{2 \sqrt{2}(\mathrm{t}-\mathrm{T})}}-1
$$

Substituting (29) into (26) we have the optimal (state) feedback control policy:

$$
\mathrm{k}(\mathrm{t})=\left[1-\sqrt{2} \frac{\sqrt{2}+1-(\sqrt{2}-1) \mathrm{e}^{2 \sqrt{2}(\mathrm{t}-\mathrm{T})}}{\sqrt{2}+1+(\sqrt{2}-1) \mathrm{e}^{2 \sqrt{2}(\mathrm{t}-\mathrm{T})}}\right] \mathrm{z}(\mathrm{t})
$$

Riccati solution by dynamic programming principle: Suppose $\tilde{w}(z, t): R^{n} \times R^{n} \rightarrow R$ is a value function whose value is the minimum value of the objective function obtained earlier (24) and (25) (choosing the same values:

$$
\mathrm{A}_{1}=-1(\text { by } \eta=1, \gamma=1), \quad \mathrm{B}_{1}=1, \mathrm{Q}=\mathrm{h}=1 \quad \text { and } \quad \mathrm{R}=\mathrm{C}=1
$$

for the inventory system given that we start it at time $\mathrm{t}$ in state $\mathrm{z}$. That is:

$$
\tilde{\mathrm{w}}(\mathrm{z}, \mathrm{t})=\inf \mathrm{J}(\mathrm{k}(\mathrm{t}))
$$

where, the value function $\tilde{\mathrm{w}}(\mathrm{z}, \mathrm{t})$ is finite valued and twice continuous differentiable on $(0, \infty) \times[0, \mathrm{~T})$ By the Principle of Optimality ${ }^{[3]}$, it is natural that $\tilde{\mathrm{w}}(\mathrm{z}, \mathrm{t})$ solves the following Hamilton Jacobi-Bellman (in short, HJB) equation:

$$
\begin{aligned}
& \tilde{\mathrm{w}}_{\mathrm{t}}(\mathrm{z}, \mathrm{t})+\min _{\mathrm{k}}\left[\mathrm{k}^{2}(\mathrm{t})+\mathrm{k}(\mathrm{t}) \tilde{\mathrm{w}}(\mathrm{z}, \mathrm{t})\right]-\mathrm{z}(\mathrm{t}) \tilde{\mathrm{w}}_{\mathrm{z}}(\mathrm{z}, \mathrm{t}) \\
& +\mathrm{v}(\mathrm{t}) \tilde{\mathrm{w}}_{\mathrm{z}}(\mathrm{z}, \mathrm{t})+\mathrm{z}^{2}(\mathrm{t})=0, \quad 0 \leq \mathrm{t}<\mathrm{T}
\end{aligned}
$$

with the terminal boundary condition $\tilde{\mathrm{w}}(\mathrm{z}, 0)=0$ and where $\tilde{\mathrm{w}}_{\mathrm{z}}(\mathrm{z}, \mathrm{t})$ and $\tilde{\mathrm{w}}_{\mathrm{t}}(\mathrm{z}, \mathrm{t})$ are the partial derivatives of $\tilde{\mathrm{w}}(\mathrm{z}, \mathrm{t})$ with respect to $\mathrm{z}$ and $\mathrm{t}$ respectively.

In order to solve the HJB Eq. 30 we minimize the expression inside the bracket of (30) and taking derivative with respect to $\mathrm{k}(\mathrm{t})$ setting it to zero. Thus the procedure yields:

$\mathrm{k}^{*}(\mathrm{t})=-\frac{1}{2} \tilde{\mathrm{w}}(\mathrm{z}, \mathrm{t})$

Substituting (31) into (30) yields the equation:

$$
\begin{aligned}
& \tilde{\mathrm{w}}_{\mathrm{t}}(\mathrm{z}, \mathrm{t})-\frac{\tilde{\mathrm{w}}_{\mathrm{z}}{ }^{2}(\mathrm{z}, \mathrm{t})}{4}-\mathrm{z}(\mathrm{t}) \tilde{\mathrm{w}}_{\mathrm{z}}(\mathrm{z}, \mathrm{t}) \\
& +\mathrm{v}(\mathrm{t}) \tilde{\mathrm{w}}_{\mathrm{z}}(\mathrm{z}, \mathrm{t})+\mathrm{z}^{2}(\mathrm{t})=0
\end{aligned}
$$


known as the HJB equation. This is a partial differential equation which has a solution form:

$\tilde{\mathrm{w}}(\mathrm{z}, \mathrm{t})=\mathrm{a}(\mathrm{t}) \mathrm{z}^{2}(\mathrm{t})$

Then:

$\tilde{\mathrm{w}}_{\mathrm{z}}(\mathrm{z}, \mathrm{t})=2 \mathrm{a}(\mathrm{t}) \mathrm{z}(\mathrm{t}), \quad \tilde{\mathrm{w}}_{\mathrm{t}}(\mathrm{z}, \mathrm{t})=\frac{\partial \mathrm{a}(\mathrm{t})}{\partial \mathrm{t}} \mathrm{z}^{2}(\mathrm{t})$

Substituting (33) and (34) into (32) yields:

$\left[1-\mathrm{a}^{2}(\mathrm{t})+2 \mathrm{a}(\mathrm{t})(\mathrm{v}(\mathrm{t})-1)+\frac{\partial \mathrm{a}(\mathrm{t})}{\partial \mathrm{t}}\right] \mathrm{z}(\mathrm{t})=0$

Since (35) must hold for any value of $z$, we must have:

$$
\frac{\partial \mathrm{a}(\mathrm{t})}{\partial \mathrm{t}}+2 \mathrm{a}(\mathrm{t})(\mathrm{v}(\mathrm{t})-1)-\mathrm{a}^{2}(\mathrm{t})+1=0
$$

is called a Riccati equation where:

$\mathrm{a}(\mathrm{t})=\mathrm{a}(0) \mathrm{e}^{-\mathrm{t}(\mathrm{v}(\mathrm{t})-1)}+\int_{0}^{\mathrm{t}} \mathrm{e}^{(\tilde{\tau}-\mathrm{t})(\mathrm{v}(\mathrm{t})-1)}\left\{\mathrm{a}^{2}(\tilde{\tau})-1\right\} \mathrm{d} \tilde{\tau}$.

Therefore, (33) is a solution form of (32). Since (33) is a solution of the HJB Eq. 32, then the optimal control $\mathrm{k}^{*}(\mathrm{t})$ can be written as $\mathrm{k}^{*}(\mathrm{t})=-\mathrm{a}(\mathrm{t}) \mathrm{z}(\mathrm{t})$, where $\mathrm{a}(\mathrm{t})$ is known constant.

An application to quadratic control theory: We study the optimal control problem (25) to minimize the production cost over all $M$ subject to an ordinary differential state equation:

$d z^{*}(t)=\left[A_{1}(t) z^{*}(t)+k^{*}(t)+v(t)\right] d t \quad z^{*}(0)=z$

where, $\mathrm{A}_{1}(\mathrm{t})=-\eta \gamma \mathrm{t}^{\gamma-1}$ and $\mathrm{M}$ denotes the class of all progressively measurable $\mathrm{F}(\mathrm{t})$ adaptive processes $\mathrm{k}(\mathrm{t})$ such that:

$\lim _{T \rightarrow \infty} \frac{1}{T} E\left[|z(t)|^{m+1}\right]=0 \quad$ for the response $z(t)$ to $k(t)$

By the same line as (30) we consider the Bellman equation associated with the problem (24):

$$
\begin{aligned}
& \tilde{\mathrm{w}}_{\mathrm{t}}(\mathrm{z}, \mathrm{t})+\min _{\mathrm{k}}\left[\mathrm{k}^{2}(\mathrm{t})+\mathrm{k}(\mathrm{t}) \tilde{\mathrm{w}}_{\mathrm{z}}(\mathrm{z}, \mathrm{t})\right]+\mathrm{A}_{1}(\mathrm{t}) \mathrm{z}(\mathrm{t}) \tilde{\mathrm{w}}_{\mathrm{z}}(\mathrm{z}, \mathrm{t}) \\
& +\mathrm{v}(\mathrm{t}) \tilde{\mathrm{w}}_{\mathrm{z}}(\mathrm{z}, \mathrm{t})+\mathrm{z}^{2}(\mathrm{t})=0, \text { if } \tilde{Q}:=(0, \infty) \times[0, T) \\
& \tilde{\mathrm{w}}(\mathrm{z}, \mathrm{T})=0, \quad \mathrm{z}>0
\end{aligned}
$$

We assume $\tilde{\mathrm{w}}(\mathrm{z}, \mathrm{t}) \in \mathrm{R}$ is a continuous, nonnegative, convex function satisfying the polynomial growth condition such that:

$0 \leq \tilde{\mathrm{w}}(\mathrm{z}) \leq \mathrm{P}\left(1+|\mathrm{z}|^{\mathrm{m}+1}\right), \quad \mathrm{z} \in \mathrm{R}, \quad \mathrm{m} \in \mathrm{N}_{+}$

for some constant $\mathrm{P}>0$

Let us choose the parameters of Weibull distribution in such way so that this distribution can be used to model decreasing rate of deterioration and then we can assume that:

$\mathrm{A}(\mathrm{t})+\mathrm{v}(\mathrm{t})<0 \quad$ for sufficiently large demand rate $\mathrm{y}(\mathrm{t})$

Lemma 1: Under (39), the differential equation:

$d z^{*}(t)=\left[A_{1}(t) z^{*}(t)+\rho\left(\tilde{w}_{z}\left(z^{*}(t)\right)\right)+v(t)\right] d t \quad z^{*}(0)=z$

admits a unique solution $\mathrm{z}^{*}(\mathrm{t})$, where:

$\rho(z)=\left\{\begin{array}{ccc}k & \text { if } & z \leq-2 k, \\ -\frac{z}{2} & \text { if } & -2 k<z \leq 0, \\ 0 & \text { if } & 0<z\end{array}\right.$

Further, for any $m \in N_{+}$, there exists $P_{1}>0$ such that:

$E\left[\left|z^{*}(t)\right|^{2 m}\right] \leq P_{1}(1+t)$

Proof: Since $\rho\left(\tilde{\mathrm{w}}_{\mathrm{z}}(\mathrm{z})\right)$ is bounded, Eq. 41 admits a unique strong solution $\mathrm{z}^{*}(\mathrm{t})$ with:

$E\left[\left|z^{*}(t)\right|^{2 n}\right]<\infty$

Using It ô's formula we have:

$$
\begin{aligned}
\left|z^{*}(t)\right|^{2 n}= & |z|^{2 n}+2 n \int_{0}^{t}\left\{A_{1}(t)+v(t)\right\}\left|z^{*}(t)\right|^{2 n} d t \\
& +2 n \int_{0}^{t} \rho\left(\tilde{w}_{z}\left(z^{*}(t)\right)\right)\left|z^{*}(t)\right|^{2 n-1} \operatorname{sgn}\left(z^{*}(t)\right) d t \\
& \leq|z|^{2 n}+2 n \int_{0}^{t}\{A(t)+v(t)\}\left|z^{*}(t)\right|^{2 n} d t \\
& +2 n \int_{0}^{t} \rho\left(\tilde{w}_{z}\left(z^{*}(t)\right)\right)\left|z^{*}(t)\right|^{2 n-1} \operatorname{sgn}\left(z^{*}(t)\right) d t,
\end{aligned}
$$


where, $\mathrm{A}(\mathrm{t})=\eta \gamma \mathrm{t}^{\gamma-1}$ is the instantaneous rate of deterioration of the on-hand inventory followed by the two-parameter Weibull distribution.

Now by (40) and taking expectation on both sides:

$$
\begin{aligned}
& E\left[\left|z^{*}(t)\right|^{2 n}\right] \leq|z|^{2 n} \\
& +2 n E\left[\int_{0}^{t} \rho\left(\tilde{w}_{z}\left(z^{*}(t)\right)\right)\left|z^{*}(t)\right|^{2 n-1} \operatorname{sgn}\left(z^{*}(t)\right) d t\right] \\
& =|z|^{2 n}+E\left[\int_{0}^{t} S^{(n)}(t) d t\right]
\end{aligned}
$$

Where:

$$
S^{(n)}(t)=2 n \rho\left(\tilde{w}_{z}\left(z^{*}(t)\right)\right)\left|z^{*}(t)\right|^{2 n-1} \operatorname{sgn}\left(z^{*}(t)\right)
$$

By (42) it is easily seen that $\mathrm{z} \rho\left(\tilde{\mathrm{w}}_{\mathrm{z}}\left(\mathrm{z}^{*}(\mathrm{t})\right)\right) \leq \mathrm{k}|\mathrm{z}|$ if $|\mathrm{z}| \geq \mathrm{b}$ for sufficiently large $\mathrm{b}>0$. Clearly :

$$
\sup _{t \rightarrow \infty} E\left[S^{(n)}(t) 1_{\left(\left|z^{*}(t)\right|<b\right)}\right]<\infty
$$

Also:

$$
\mathrm{E}\left[\mathrm{S}^{(\mathrm{n})}(\mathrm{t}) 1_{\left(\mathrm{z}^{*}(\mathrm{t}) \mid \geq \mathrm{b}\right)}\right] \leq \mathrm{E}\left[\mathrm{k}|\mathrm{z}| 1_{\left(\mathrm{z}^{*}(\mathrm{t}) \mid \geq \mathrm{b}\right)}\right]
$$

In addition, by (44) we see that the right-hand side of equation (45) is bounded from above. This completes the proof.

Theorem 2: We assume (39). Then the optimal control $\mathrm{k}^{*}(\mathrm{t})$ is given by:

$\mathrm{k}^{*}(\mathrm{t})=\rho\left(\tilde{\mathrm{w}}_{\mathrm{z}}\left(\mathrm{z}^{*}(\mathrm{t})\right)\right)$

Proof: Let us note that $-\frac{z^{2}}{4}=\min _{k}\left\{k^{2}+z k\right\}$ and the minimum is attained by $\rho(z)$. We apply It $\hat{o}^{\prime} \mathrm{s}$ formula for convex functions ${ }^{[17]}$ to obtain:

$$
\begin{aligned}
\tilde{\mathrm{w}}\left(\mathrm{z}^{*}(\mathrm{~T})\right)= & \tilde{\mathrm{w}}(\mathrm{z})+\int_{0}^{\mathrm{T}}\left[\left\{\mathrm{A}_{1}(\mathrm{t}) \mathrm{z}^{*}(\mathrm{t})+\mathrm{k}^{*}(\mathrm{t})+\mathrm{v}(\mathrm{t})\right\} \tilde{\mathrm{w}}_{\mathrm{z}}\left(\mathrm{z}^{*}(\mathrm{t})\right)\right. \\
& \left.+\tilde{\mathrm{w}}_{\mathrm{t}}\left(\mathrm{z}^{*}(\mathrm{t})\right)\right] \mathrm{dt}
\end{aligned}
$$

By virtue of (38)

$$
E\left[\tilde{w}\left(z^{*}\left(T \wedge \tau_{n}\right)\right)\right]=\tilde{w}(z)-E\left[\int_{0}^{T \wedge \tau_{n}}\left\{z^{*}(t)^{2}+k^{*}(t)^{2}\right\} d t\right]
$$

where, $\left\{\tau_{n}\right\}$ is a sequence of localizing stopping times for the local martingale.

By (39) and (43) of Lemma 1, we have:

$$
\begin{aligned}
& \mathrm{E}\left[\tilde{\mathrm{w}}\left(\mathrm{z}^{*}(\mathrm{t})\right)\right] \leq \mathrm{P}\left(1+\mathrm{E}\left[\left|\mathrm{Z}^{*}(\mathrm{t})\right|^{\mathrm{m}+1}\right]\right) \\
& \leq \mathrm{P}\left(1+\mathrm{E}\left[\left|\mathrm{z}^{*}(\mathrm{t})\right|^{2(\mathrm{~m}+1)}\right]^{\frac{1}{2}}\right) \\
& \leq \mathrm{P}\left(1+\left(\mathrm{P}_{1}(1+\mathrm{t})\right)^{\frac{1}{2}}\right)<\infty .
\end{aligned}
$$

Dividing both sides by $\mathrm{T}$ and letting $\mathrm{T} \rightarrow \infty$ we get:

$$
\liminf _{\mathrm{T} \rightarrow \infty} \frac{1}{\mathrm{~T}} \mathrm{E}\left[\tilde{\mathrm{w}}\left(\mathrm{z}^{*}(\mathrm{t})\right)\right]=0
$$

Hence $\mathrm{z}^{*}(\mathrm{t})$ satisfies (37). Letting $\mathrm{n} \rightarrow \infty$ to (47) and using (49) we obtain:

$$
E\left[\int_{0}^{T}\left\{z^{*}(t)^{2}+k^{*}(t)^{2}\right\} d t\right] \leq \tilde{w}(z)
$$

from which $\mathrm{J}\left(\mathrm{k}^{*}\right) \leq \tilde{\mathrm{w}}(\mathrm{z})$. Now by (48) we have $\mathrm{J}\left(\mathrm{k}^{*}\right) \leq \tilde{\mathrm{w}}(\mathrm{z})<\infty$ hence, $\mathrm{k}^{*}=\left(\mathrm{k}^{*}(\mathrm{t})\right) \in \mathrm{M}$.

Let $\mathrm{k} \in \mathrm{M}$ be arbitrary. By the same line as above, we have:

$$
\mathrm{E}[\tilde{\mathrm{w}}(\mathrm{z}(\mathrm{t}))] \geq \tilde{\mathrm{w}}(\mathrm{z})-\mathrm{E}\left[\int_{0}^{\mathrm{T}}\left\{\mathrm{z}(\mathrm{t})^{2}+\mathrm{k}(\mathrm{t})^{2}\right\} \mathrm{dt}\right], \quad \mathrm{k} \in \mathrm{M}
$$

By (39) and (37):

$$
\liminf _{\mathrm{T} \rightarrow \infty} \frac{1}{T} E[\tilde{w}(z(t))]=0
$$

Thus we can obtain the desired result.

\section{CONCLUSION}

This study has described the solution of an inventory-production system with Weibull distribution deteriorating items using both Pontryagin maximum principle and Dynamic programming principle. The resulting production control policy has minimized the objective function of the total cost with the application to quadratic control theory. These models can be extended in many ways. For example, if other costs such as the storage cost are included; or instead of 
minimizing the total cost, one may want to maximize the total profit where the unit revenue rate is both function of time and of the inventory level. However, we need to give numerical illustrative examples for this optimal control of a production-inventory system with Weibull distribution deteriorating items.

\section{ACKNOWLEDGEMENT}

The researchers wish to acknowledge the support provided by Fundamental Research Grant Scheme, No. 203/PJJAUH/671128, Universiti Sains Malaysia, Penang, Malaysia for conducting this research.

\section{REFERENCES}

1. Andijani, A. and M. AL-Dajani, 1998. Analysis of deteriorating inventory-production systems using a linear quadratic regulator. Eur. J. Operat. Res., 106: 82-89.

http://cat.inist.fr/?aModele $=$ afficheN\&cpsidt $=2222$ 563

2. Bahari-Kasani, H., 1989. Replenishment schedule for deteriorating items with time-proportional demand. J Operat. Res. Soc., 40: 75-81. http://www.jstor.org/stable/2583079

3. Bellman, R., 1957. Dynamic Programming. Princeton. Princeton University Press, New Jersey.

4. Bensoussan, A., Jr. E.G. Hurst and B. Naslund, 1974. Management Applications of Modern Control Theory. Elsevier, New York, ISBN: 0720433002, pp: 346.

5. Chakrabarty, T., B.C. Giri and K.S. Chaudiri, 1998. An EOQ model for items with Weibull distribution deterioration, shortages and trended demand: An extension of Philip's model. Comput. Operat. Res., 25: 649-657.

http://cat.inist.fr/?aModele $=$ afficheN\&cpsidt $=1636$ 909

6. Chen, J.M. and S.C. Lin. 2003. Optimal replenishment scheduling for inventory items with Weibull distributed deterioration and time-varying demand. Inform. Optimiz. Sci., 24: 1-21.

7. Covert, R.P. and G.C. Philip, 1973. An EOQ model for items with Weibull distributed deterioration. AIIE. Trans., 5: 323-326. DOI: 10.1080/05695557308974918

8. Feichtinger, G. and E.J. Dockner, 1985. Optimal pricing and production in an inventory model. Eur. J. Operat. Res., 19: 45-56. http://ideas.repec.org/a/eee/ejores/v19y1985i1p4556.html
9. Gaimon, C., 1988. Simultaneous and dynamic price, production, inventory and capacity decisions. Eur. J. Operat. Res., 35: 426-441. http://ideas.repec.org/a/eee/ejores/v35y1988i3p426 $-441 . h t m l$

10. Ghare, P.N. and G.F. Schrader, 1963. A model for exponentially decaying inventories. J. Ind. Eng., 15: 238-243.

11. Ghosh, S.K. and K.S. Chaudhuri, 2004. An orderlevel inventory model for a deteriorating item with Weibull distribution deterioration, time-quadratic demandand shortages. Adv. Model. Optimiz., 6: 21-35.

http://www.ici.ro/camo/journal/vol6/v6a2.pdf

12. Goel, V.P. and S.P. Aggarwal, 1980. Pricing and ordering policy with general Weibull rate of deteriorating inventory. Indian Journal of Pure and Applied Mathematics, 11: 618-627. http://www.new.dli.ernet.in/rawdataupload/upload/ insa/INSA_2/20005a21_618.pdf

13. Goyal, S.K. and B.C. Giri, 2001. Recent trends in modeling of deteriorating inventory. Eur. J. Operat. Res., 134: 1-16. http://ideas.repec.org/a/eee/ejores/v134y2001i1p116.html

14. Happing, U. and H. Wang, 1990. An economic ordering policy model for deteriorating items with time proportional demand. Eur. J. Operat. Res., 46: 21-27.

http://ideas.repec.org/a/eee/ejores/v46y1990i1p2127.html

15. Harlt, R.F. and S.P. Sethi, 1984. Optimal control problems with differential inclusions: Sufficiency conditions and an application to a production inventory model. Optimal Control Appli. Method., 5: 289-307. http://prolog.univie.ac.at/cgibin/pubs_abstract.cgi?pubnr $=110$

16. Hwang, C.L., L.T. Fan and L.E. Erickson, 1967. Optimal production planning by the maximum principle. Manage. Sci., 13: 751-755. http://www.jstor.org/pss/2628091

17. Karatzas, I. and S.E. Shreve, 1991. Brownian Motion and Stochastic Calculus. Springer-Verlag, New York, ISBN: 0387976558, pp: 470.

18. Misra, R.B., 1975. Optimum production lot size model for a system with deteriorating inventory. Int. J. Prod. Res., 13: 495-505. DOI: 10.1080/00207547508943019

19. Pekelman, D., 1974. Simultaneous priceproduction decision. Operat. Res., 22: 788-794. http://or.journal.informs.org/cgi/content/abstract/22 /4/788 
20. Philip, G.C., 1974. A generalized EOQ model for items with weibull distribution. AIIE. Trans., 6: 159-162. DOI: $10.1080 / 05695557408974948$

21. Pontrygin, L.S., V.G. Boltyanski, R.V. Gamkrelidge and E.F. Mischenko, 1962. The Mathematical Theory of Optimal Processes. 6th Edn., John Wiley and Sons, New York, pp: 360. http://books.google.com.pk/books?id=udNSAAAA MAAJ\&q $=$ The + mathematical + theory + of + optimal + processes\&dq $=$ The + mathematical + theory + of + opt imal + processes\&pgis $=1$

22. Sethi, S.P. and G.L. Thompson, 2000. Optimal Control Theory, Applications to Management Science and Economics. 2nd Edn., Springer, USA., ISBN: 0792386086, pp: 504.
23. Shah, N. H. and Y.K. Shah, 2000. Literature survey on inventory model for deteriorating items. Econ. Ann., 44: 221-237. http://scindeks.nb.rs/article.aspx?artid=001332640005221S\&lang=en

24. Sprzeuzkouski, A.Y., 1967. A problem in optimal stock management. J. Optim. Theor. Appli., 1: 232-241. DOI: $10.1007 / \mathrm{BF} 00926065$.

25. Wu, J.W. and W.C. Lee, 2003. An EOQ inventory model for items with Weibull distributed deterioration, shortages and time-varying demand. Inform. Optimiz. Sci., 24: 103-122. 